A Jacobi-Davidson type projection method for nonlinear eigenvalue problems

Betcke, Timo and Voss, Heinrich

2004

MIMS EPrint: 2006.391

Manchester Institute for Mathematical Sciences

School of Mathematics

The University of Manchester

\footnotetext{
Reports available from: http://eprints.maths.manchester.ac.uk/

And by contacting: The MIMS Secretary

School of Mathematics

The University of Manchester

Manchester, M13 9PL, UK
} 


\title{
A Jacobi-Davidson-type projection method for nonlinear eigenvalue problems
}

\author{
Timo Betcke ${ }^{\mathrm{a}}$ Heinrich Voss ${ }^{\mathrm{b}, 1}$ \\ ${ }^{a}$ Oxford University Computing Laboratory, Wolfson Building, Parks Road, Oxford, \\ OX13QD, England, timo.betcke@comlab.ox.ac.uk \\ b Technical University of Hamburg-Harburg, Department of Mathematics, \\ D-21071 Hamburg, Federal Republic of Germany, voss@tu-harburg.de
}

\begin{abstract}
This article discusses a projection method for nonlinear eigenvalue problems. The subspace of approximants is constructed by a Jacobi-Davidson type approach, and the arising eigenproblems of small dimension are solved by safeguarded iteration. The method is applied to a rational eigenvalue problem governing the vibrations of tube bundle immersed in an inviscid compressible fluid.
\end{abstract}

Key words: nonlinear eigenvalue problem, Jacobi-Davidson method, projection method, Rayleigh functional, minmax characterization PACS: 02.60.Dc

\section{Introduction}

In this paper we consider the nonlinear eigenvalue problem

$$
T(\lambda) x=0
$$

where $T(\lambda) \in \mathbb{R}^{n \times n}, \lambda \in J$, is a family of real symmetric matrices, and $J \subset \mathbb{R}$ is an open interval. As in the linear case, $\lambda \in J$ is called an eigenvalue if equation (1) has a nontrivial solution $x \neq 0$, and $x$ is called a corresponding eigenvector. Typically, we assume that $n$ is large, and $T(\lambda)$ is sparse.

$\overline{1}$ Corresponding author: Email: voss@tu-harburg.de, Phone: +49 4042878 3279, Fax: +494042878 2696 
For linear sparse eigenproblems $T(\lambda)=\lambda B-A$ the most efficient methods (Lanczos method, Arnoldi method, Jacobi-Davidson method, e.g.) are iterative projection methods, where approximations to the wanted eigenvalues and eigenvectors are obtained from projections of the eigenproblem to subspaces of small dimension which are expanded in the course of the algorithm.

Ruhe [9] and Hager and Wiberg [4], [5] suggested a generalization of this approach to nonlinear eigenvalue problems. Similarly as in the rational Krylov process they construct a sequence $V_{k}$ of subspaces of $\mathbb{R}^{n}$ and corresponding Hessenberg matrices $H_{k}$ which approximate the projection of $T(\sigma)^{-1} T\left(\lambda_{k}\right)$ to $V_{k}$. Here $\sigma$ denotes a shift and $\lambda_{k}$ an approximation to the wanted eigenvalue of (1). Then a Ritz vector of $H_{k}$ corresponding to an eigenvalue of small modulus approximates an eigenvector of the nonlinear problem from which a (hopefully) improved eigenvalue approximation of problem (1) is obtained.

In this paper we propose a further projection method for problem (1). We do not construct a sequence of linear approximations of the nonlinear problem but we update projections of the nonlinear problem to a sequence of nested subspaces of small dimension which are expanded in a similar way as in the Jacobi-Davidson method. Differently from Ruhe's approach the projected problems inherit the symmetry from the original problem (1). Moreover, if the eigenvalues of problem (1) can be characterized as minmax values of a Rayleigh functional then the same holds for the eigenvalues of the projected problem, and it can be solved efficiently by the safeguarded iteration method.

Our paper is organized as follows. Section 2 summarizes minmax properties for nonlinear eigenvalue problems, and Section 3 briefly reviews numerical methods for finite dimensional nonlinear problems. Section 4 introduces the modification of the Jacobi-Davidson method for nonlinear eigenvalue problems. It discusses the solution of the correction equation and a restart procedure to keep the storage requirements at a reasonable size and which can be used to purge unwanted directions as well. In Section 5 we report on our numerical experience for a rational eigenvalue problem governing the the vibrations of a tube bundle immersed in an inviscid compressible fluid.

\section{Minmax characterization of eigenvalues}

We consider the nonlinear eigenvalue problem $T(\lambda) x=0$ where $T(\lambda) \in \mathbb{R}^{n \times n}$ is a family of real symmetric matrices for every $\lambda$ in an open real interval $J$ which may be unbounded.

For a linear symmetric problem $A x=\lambda x$ all eigenvalues are real, and if they are ordered by magnitude regarding their multiplicities $\lambda_{1} \leq \lambda_{2} \leq \ldots \leq \lambda_{n}$ 
then it is well known that they can be characterized by the minmax principle of Poincaré or by the maxmin principle of Courant and Fischer.

Similar results hold for certain nonlinear eigenvalue problems, too. We assume that the function $f(\lambda, x):=x^{T} T(\lambda) x$ is continuously differentiable on $J \times \mathbb{R}^{n}$, and that for every fixed $x \in \mathbb{R}^{n} \backslash\{0\}$ the real equation

$$
f(\lambda, x)=0
$$

has at most one solution in $J$. Then equation (2) implicitly defines a functional $p$ on some subset $D$ of $\mathbb{R}^{n} \backslash\{0\}$ which replaces the Rayleigh quotient in the variational characterization of eigenvalues of problem (1), and which we call the Rayleigh functional.

Moreover, we assume that

$$
\left.\frac{\partial}{\partial \lambda} f(\lambda, x)\right|_{\lambda=p(x)}>0 \quad \text { for every } x \in D
$$

For nonlinear eigenvalue problems variational properties using the Rayleigh functional were proved by Duffin, Rogers, Hadeler, and Werner for overdamped problems, i.e. if the Rayleigh functional $p$ is defined in the entire space $\mathbb{R}^{n} \backslash\{0\}$. Nonoverdamped problems were studied by Barston for quadratic problems, and by Werner and the second author for general problems (c.f. [15] and the literature given therein).

In the general case the natural enumeration for which the smallest eigenvalue is the first one, the second smallest is the second one, etc. is not appropriate, but the number of an eigenvalue $\lambda$ of the nonlinear problem (1) is inherited from the location of the eigenvalue 0 in the spectrum of the matrix $T(\lambda)$.

If $\lambda \in J$ is an eigenvalue of problem (1) then $\mu=0$ is an eigenvalue of the linear problem $T(\lambda) y=\mu y$. Since $T(\lambda)$ is a symmetric matrix, all eigenvalues are real and can be ordered by magnitude regarding their multiplicity $\mu_{1} \geq \mu_{2} \geq \ldots$. In particular, $\mu_{k}=0$ for some $k \in \mathbb{N}$, i.e. 0 is the $k$ largest eigenvalue. In this case we call $\lambda$ a $k$-th eigenvalue of (1).

With this enumeration the following minmax characterization of the eigenvalues of the nonlinear eigenproblem (1) was proved in [15] (under an additional compactness condition even for the infinite dimensional case):

Theorem 1: Under the conditions given above the following assertions hold:

(i) For every $k \in \mathbb{N}$ there is at most one $k$-th eigenvalue of problem (1) which 
can be characterized by

$$
\lambda_{k}=\min _{\substack{V \in S_{k} \\ V \cap D \neq \emptyset}} \max _{v \in V \cap D} p(v) .
$$

(ii) If

The set of eigenvalues of (1) in $J$ is at most countable.

$$
\lambda_{k}=\inf _{\substack{V \in S_{k} \\ V \cap D \neq \emptyset}} \sup _{v \in V \cap D} p(v) \in J
$$

for some $k \in \mathbb{N}$ then $\lambda_{k}$ is the $k$-th eigenvalue of (1) and the inf and sup are attained by some $V \in S_{k}$ and some $v \in V \cap D$, i.e. (3) holds.

(iii) If for $k<\ell$ there exist a $k$-th and an $\ell$-th eigenvalue $\lambda_{k}$ and $\lambda_{\ell}$ in $J$, then $J$ contains an $m$-th eigenvalue $\lambda_{m}$ for $m=k, \ldots, \ell$, and $\lambda_{k} \leq \lambda_{k+1} \leq \ldots \lambda_{\ell}$.

\section{Solving nonlinear eigenvalue problems}

In this section we briefly review numerical methods for finite dimensional nonlinear eigenvalue problems $T(\lambda) x=0$, where $T(\lambda)$ is a family of real symmetric $n \times n$-matrices. We only consider methods for the general problem (1), and do not take into account the rich literature on quadratic or polynomial $\lambda$-matrices.

For dense problems algorithms are investigated in [6], [8] and [13] which are all variants of inverse iteration

$$
x^{k+1}=\alpha_{k} T\left(\sigma_{k}\right)^{-1} T^{\prime}\left(\sigma_{k}\right) x^{k} .
$$

Here $\alpha_{k}$ is a suitable normalization factor, and $\sigma_{k}$ is updated in some way. Similarly as in the linear case inverse iteration converges locally. The convergence is quadratic for simple eigenvalues, and it is even cubic if $T(\lambda)$ is symmetric, and $\sigma_{k}$ is updated by the Rayleigh functional.

Moreover, under the conditions of Section 2 inverse iteration can be safeguarded in a similar way as for linear eigenproblems. Assume that problem (1) has an $m$-th eigenvalue $\lambda_{m} \in J$, and let $T(\lambda)=L(\lambda) D(\lambda) L(\lambda)^{T}$ be an $L D L^{T}$-factorization of $T(\lambda)$ for some $\lambda \in J$. If the number $d^{+}(\lambda)$ of positive diagonal elements of $D(\lambda)$ is less than $m$, then it can be shown that $\lambda<\lambda_{m}$, and if $d^{+}(\lambda) \geq m$ then $\lambda \geq \lambda_{m}$ (cf. [15]).

An essential disadvantage of inverse iteration is the fact that each eigenvalue has to be determined individually by an iterative process, and that each step of this iteration requires the solution of a linear system. Moreover, the coefficient matrix $T\left(\sigma_{k}\right)$ of system (1) changes in each step. In contrast to the linear case 
replacing (4) by $x^{k+1}=\alpha_{k} T(\tilde{\sigma})^{-1} T^{\prime}\left(\sigma_{k}\right) x^{k}$ with a fixed shift $\tilde{\sigma}$ results in misconvergence. It is easily seen that this iteration converges to an eigenpair of the linear problem $T(\tilde{\sigma}) x=\gamma T^{\prime}(\tilde{\lambda}) x(\gamma \neq 0$ and $\tilde{\lambda}$ depending on the normalization condition) from which we can not recover an eigenpair of the nonlinear problem (1).

A remedy against this wrong convergence was proposed by Neumaier [6] who introduced the so called residual inverse iteration which converges linearly with a fixed shift, and quadratically or cubically if the coefficient matrix is altered in every iteration step according to reasonable updates of $\sigma_{k}$.

For symmetric problems satisfying the conditions of Section 2 the following method called safeguarded iteration was introduced in [13]. Assume that we are given an approximation $\sigma_{k} \in J$ to an $m$-th eigenvalue of problem (1). Determine an eigenvector $x_{k}$ corresponding to the $m$-largest eigenvalue of the matrix $T\left(\sigma_{k}\right)$, and evaluate $\sigma_{k+1}:=p\left(x_{k}\right)$. This method converges locally to an $m$-th eigenvalue $\lambda_{m} \in J$, and the convergence is quadratic. For positive definite $T^{\prime}(\lambda)$ for $\lambda \in J$ it is even cubic, if $x_{k}$ is replaced by an eigenvector of the general eigenvalue problem $T\left(\sigma_{k}\right) x=\mu_{k} T^{\prime}\left(\sigma_{k}\right) x$ corresponding to the $m$-largest eigenvalue $\mu_{k}$. The method is closely related to inverse iteration (4) (and for small dimensions it is of equal cost), namely $x_{k+1}$ in (4) is exactly the first iterate of inverse iteration for the linear eigenproblem $T\left(\sigma_{k}\right) x=\mu_{k} T^{\prime}\left(\sigma_{k}\right) x$ with initial guess $x_{k}$.

For dense problems inverse iteration and its variants mentioned above are very capable methods, however, for large and sparse nonlinear eigenvalue problems inverse iteration is much too expensive.

For sparse linear eigenvalue problems the most efficient methods are iterative projection methods, where approximations of the wanted eigenvalues and corresponding eigenvectors are obtained from projections of the eigenproblem to subspaces which are expanded in the course of the algorithm. Methods of this type for symmetric problems are the Lanczos method, rational Krylov subspace methods, and the Jacobi-Davidson method, e.g. (cf. [1]).

In some sense, Ruhe [9] and Hager and Wiberg [5], [4] generalized this approach to sparse nonlinear eigenvalue problems by nesting the linearization of problem (1) by Regula falsi and the solution of the resulting linear eigenproblem by Arnoldi's method, where the Regula falsi iteration and the Arnoldi recursion are knit together. Similarly as in the rational Krylov process they construct a sequence $V_{k}$ of subspaces of $\mathbb{R}^{n}$ and corresponding Hessenberg matrices $H_{k}$ which approximate the projection of $T(\sigma)^{-1} T\left(\lambda_{k}\right)$ to $V_{k}$. Here $\sigma$ denotes a shift and $\lambda_{k}$ an approximation to the wanted eigenvalue of (4). Then a Ritz vector of $H_{k}$ corresponding to an eigenvalue of small modulus approximates an eigenvector of the nonlinear problem from which a (hopefully) improved 
eigenvalue approximation of problem (1) is obtained.

In [4] Hager points out that the eigenvalues and eigenvectors are determined one after another. After a Ritz value has converged only the approximate eigenvectors from previous Arnoldi runs, the just converged Ritz vector, and an approximation to a further eigenvector to be computed in the next Arnoldi run are kept, and the rest of the current Krylov space is purged. Hence, each eigenvalue of (1) is determined by an individual approximate Arnoldi process essentially from scratch, and therefore the cost of the rational Krylov method for nonlinear problems is similar to the cost of inverse iteration.

A further severe drawback of the approach of Ruhe, Hager and Wiberg is the fact that one does not take advantage of symmetry properties of the nonlinear problem (1).

\section{A Jacobi-Davidson type projection method}

In this section we describe an algorithm which combines the fast convergence of safeguarded iteration with the efficiency of iterative projection methods. It is strongly related to the Jacobi-Davidson method for linear eigenvalue problems.

\subsection{The Jacobi-Davidson Method for Linear Eigenvalue Problems}

The Jacobi-Davidson method introduced by Sleijpen and van der Vorst (cf. [10], [11]) for the linear eigenvalue problem

$$
A x=\lambda x
$$

is an iterative projection method. If $V$ is a matrix with orthonormal columns, and $\left(\sigma_{k}, v_{k}\right)$ is an eigenpair of the projected problem $V^{T} A V v=\sigma v$, then the corresponding Ritz pair $\left(\sigma_{k}, u_{k}\right), u_{k}=V v_{k}$, approximating an eigenpair of (5) is improved as follows. The matrix $V$ is expanded by an orthogonal correction $t$ of $u_{k}$, and $V$ is replaced by $[V, t]$.

The most desirable orthogonal correction $t$ solves the equation

$$
A\left(u_{k}+t\right)=\lambda\left(u_{k}+t\right), \quad t \perp u_{k} .
$$


As $t \perp u_{k}$ the operator $A$ can be restricted to the subspace orthogonal to $u_{k}$ yielding $\left(I-u_{k} u_{k}^{T}\right) A\left(I-u_{k} u_{k}^{T}\right)$, and (6) can be rewritten as

$$
\left(I-u_{k} u_{k}^{T}\right)(A-\lambda I)\left(I-u_{k} u_{k}^{T}\right) t=-\left(A-\sigma_{k} I\right) u_{k} .
$$

Here we assumed that $u_{k}$ is normalized by $\left\|u_{k}\right\|=1$.

Approximating the unknown $\lambda$ by $\sigma_{k}$ we finally arrive at the Jacobi-Davidson correction equation for the update $t \perp u_{k}$ :

$$
\left(I-u_{k} u_{k}^{T}\right)\left(A-\sigma_{k} I\right)\left(I-u_{k} u_{k}^{T}\right) t=-r_{k}
$$

where $r_{k}:=\left(A-\sigma_{k} I\right) u_{k}$ denotes the residual of $\left(\sigma_{k}, u_{k}\right)$.

Implementation details of the Jacobi-Davidson method for various types of eigenvalue problems can be found in [1]. In particular, numerical experiments show (cf. [12]) that the correction equation only needs to be solved approximately. Normally only a small number of steps of a preconditioned Krylov subspace method are sufficient to obtain a good expansion $t$ for the subspace $V$.

\subsection{Modifications for Nonlinear Eigenvalue Problems}

To extend the idea of the Jacobi-Davidson method to nonlinear eigenvalue problems of type (1) we use the correction equation

$$
\left(I-\frac{p_{k} u_{k}^{T}}{u_{k}^{T} p_{k}}\right) T\left(\sigma_{k}\right)\left(I-\frac{u_{k} u_{k}^{T}}{u_{k}^{T} u_{k}}\right) t=-r_{k}, \quad t \perp u_{k},
$$

where $p_{k}:=T^{\prime}\left(\sigma_{k}\right) u_{k}$ and $r_{k}:=T\left(\sigma_{k}\right) u_{k}$, and $\left(\sigma_{k}, u_{k}\right)$ is the current approximation to an eigenpair of $T(\lambda) x=0$ obtained from a projected problem

$$
V^{T} T(\lambda) V v=0, \quad u=V v .
$$

Obviously, equation (8) is equivalent to

$$
T\left(\sigma_{k}\right) t-\alpha p_{k}=-r_{k},
$$

where $\alpha$ has to be chosen such that $t \perp u_{k}$. Solving for $t$ we obtain

$$
t=-u_{k}+\alpha T\left(\sigma_{k}\right)^{-1} p_{k}=-u_{k}+\alpha T\left(\sigma_{k}\right)^{-1} T^{\prime}\left(\sigma_{k}\right) u_{k},
$$

and since $u_{k} \in V$ it follows that $\operatorname{span}\{V, t\}=\operatorname{span}\{V, \tilde{t}\}$ where

$$
\tilde{t}=\alpha T\left(\sigma_{k}\right)^{-1} T^{\prime}\left(\sigma_{k}\right) u_{k} .
$$


Hence, the space spanned by the columns of $V$ is expanded by the direction obtained from inverse iteration, which converges cubically if $\sigma_{k}$ is chosen as Rayleigh-Functional of $u_{k}$. Thus, cubic convergence can be expected if equation (8) is solved exactly. Numerical experiments have shown that even a moderate approximate solution of (8) with some steps of preconditioned GMRES also leads to fast convergence which is nearly cubic.

The view of Jacobi-Davidson as accelerated inverse iteration can be found in [10] for the linear case. Equation (8) is already used in [2] for quadratic eigenvalue problems. There the projected problem is solved by linearization of the projected quadratic problem. Our approach is to combine the correction equation (8) with safeguarded iteration for the projected problem to solve nonlinear eigenvalue problems of type (1) satisfying the conditions of Section 2. The resulting method is given in Algorithm 1.

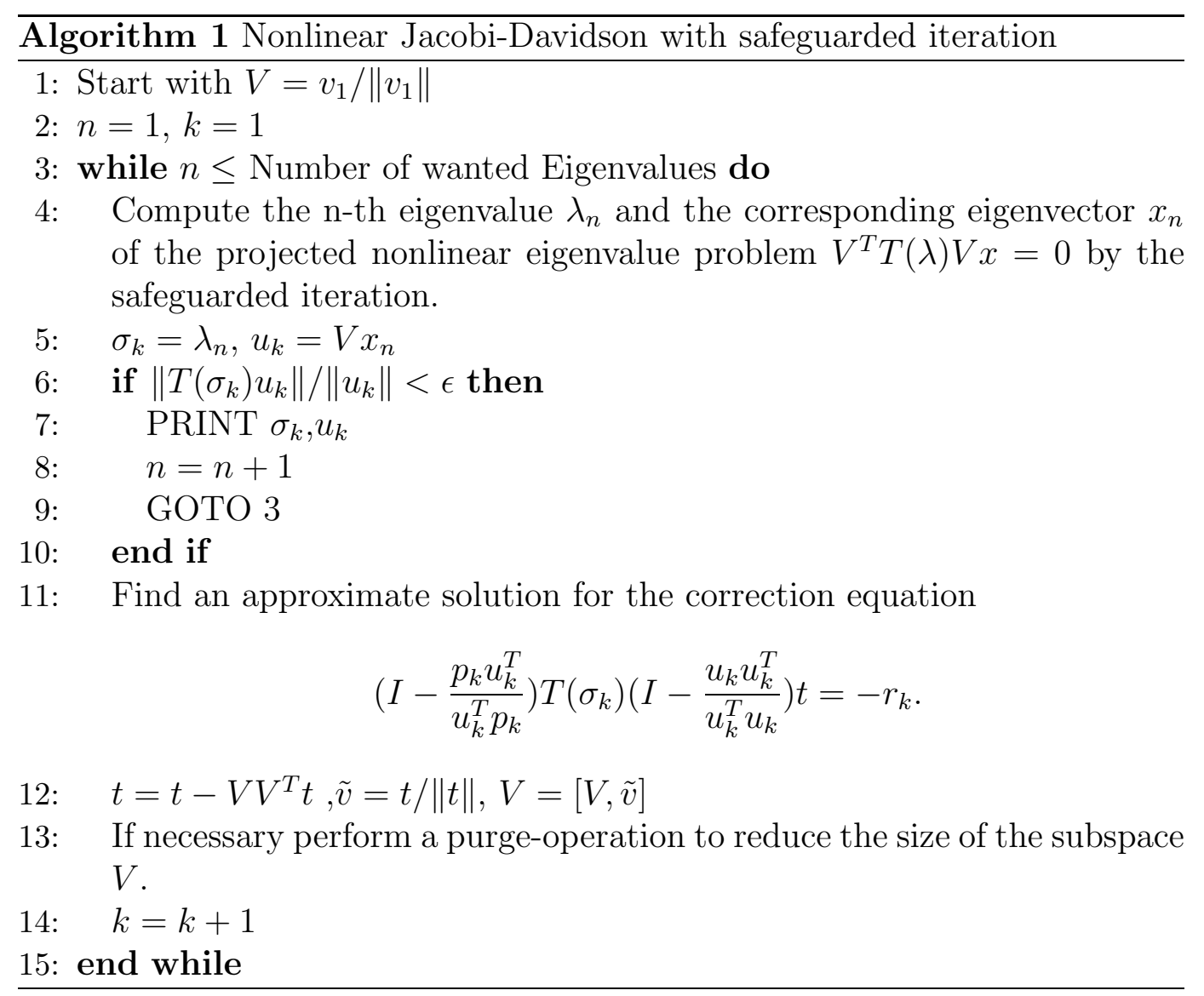

\section{Remarks}

1-2: $v_{1}$ is an initial approximation to the eigenvector corresponding to the first eigenvalue of (1). If the algorithm shall not start to iterate for the first eigenvalue of (1), but for the $\mathrm{k}$-th eigenvalue, then a suitable k-dimensional start space must be given to the algorithm.

4-5: Here the safeguarded iteration for nonlinear eigenvalue problems, which is 
described in Section 3 is used. It can be performed with low cost because the dimension of the projected problem is small.

11: Will be discussed in Subsection 4.3

12: Due to the better numerical stability it is preferable to use modified GramSchmidt in implementations of the algorithm.

13: See Subsection 4.4

\subsection{Solving the correction equation}

The correction equation (8) is solved approximately with some steps of GMRES with an appropriate preconditioner $K$ for $T\left(\sigma_{k}\right)$.

The operator $T\left(\sigma_{k}\right)$ is restricted to map the subspace $\left\{u_{k}\right\}^{\perp}$ to $\left\{T^{\prime}\left(\sigma_{k}\right) u_{k}\right\}^{\perp}$ in the correction equation (8). Therefore, the preconditioner also has to be modified, and instead of $K$ we use the preconditioner

$$
\tilde{K}:=\left(I-\frac{p_{k} u_{k}^{T}}{u_{k}^{T} p_{k}}\right) K\left(I-\frac{u_{k} u_{k}^{T}}{u_{k}^{T} u_{k}}\right)
$$

for the restricted operator

$$
\tilde{T}\left(\sigma_{k}\right):=\left(I-\frac{p_{k} u_{k}^{T}}{u_{k}^{T} p_{k}}\right) T\left(\sigma_{k}\right)\left(I-\frac{u_{k} u_{k}^{T}}{u_{k}^{T} u_{k}}\right) .
$$

With left-preconditioning equation (8) becomes

$$
\tilde{K}^{-1} \tilde{T}\left(\sigma_{k}\right) t=-\tilde{K}^{-1} r_{k}, \quad t \perp u_{k}
$$

We apply a Krylov solver to equation (10) with initial guess $t=0$. For the linear case this was already discussed in [12], and the transfer to equation (10) is straight forward.

Since the operator $\tilde{K}^{-1} \tilde{T}\left(\sigma_{k}\right)$ maps the space $\left\{u_{k}\right\}^{\perp}$ into itself all iterates are contained in $\left\{u_{k}\right\}^{\perp}$, and therefore in each step we have to perform the matrix-vector product

$$
y=\tilde{K}^{-1} \tilde{T}\left(\sigma_{k}\right) v
$$

for some $v \in\left\{u_{k}\right\}^{\perp}$.

This can be done in 2 steps. First multiply $v$ by $\tilde{T}\left(\sigma_{k}\right)$ which yields

$$
\tilde{y}=\left(I-\frac{p_{k} u_{k}^{T}}{u_{k}^{T} p_{k}}\right) T\left(\sigma_{k}\right) v,
$$


and then solve

$$
\tilde{K} y=\tilde{y}, \quad y \perp u_{k} .
$$

This equation can be rewritten as

$$
K y-\alpha p_{k}=\tilde{y}
$$

where $\alpha$ is determined from the condition that $y \perp u_{k}$. Thus, we finally obtain

$$
y=K^{-1} \tilde{y}-\frac{u_{k}^{T} K^{-1} \tilde{y}}{u_{k}^{T} K^{-1} p_{k}} K^{-1} p_{k}
$$

To conclude, the approximate solution of the the linear system (8) by a preconditioned Krylov solver requires one matrix-vector product to get $K^{-1} p_{k}$, and one more product in each step to obtain $K^{-1} \tilde{y}$. Therefore, taking into account the projections in the correction equation raises the number of matrix-vector multiplications only by one.

\subsection{Restarts}

As the subspaces expand in the course of the algorithm the increasing storage or the computational overhead may make it necessary to restart and purge some of the basis vectors. An obvious way to restart motivated by the linear theory is to take an orthonormal basis of the space spanned by the eigenvectors of (1) already computed. However, for nonlinear problems this space is not appropriate.

The proof of the minmax characterization

$$
\lambda_{\ell}=\min _{\substack{W \in S_{\ell} \\ W \cap D \neq \emptyset}} \max _{w \in W \cap D} p(w)
$$

in [15] shows that the minimum is attained by the invariant subspace $W$ of $T\left(\lambda_{\ell}\right)$ corresponding to the $\ell$ largest eigenvalues. Hence, if $W$ denotes an orthonormal basis of $W$ as well the $\ell$-th eigenvalue of the projected problem

$$
W^{T} T(\lambda) W x=0
$$

is $\lambda_{\ell}$, and $W$ or any subspace of $\mathbb{R}^{n}$ containing $W$ yields a perfect restart.

If $\sigma_{k}$ is the current approximation to $\lambda_{\ell}$ obtained from the projection of (1) using the matrix $V$ with orthonormal columns we therefore determine the matrix $S$ of eigenvectors corresponding to the $\ell$ largest eigenvalues of

$$
V^{T} T\left(\sigma_{k}\right) V x=\mu x
$$


and continue with the reduced basis $\tilde{V}=V S$. If we restart with this $\ell$ dimensional space we may cut off valuable information about higher eigenvalues that is contained in the discarted part of the subspace $V$. Actually in one of our examples we observed that it took unusually many steps to determine the next eigenvalue after a restart. We therefore continued with $\tilde{\ell}$ eigenvectors of (13) where $\tilde{\ell}$ is slightly larger than $\ell$, say $\tilde{\ell}=\ell+3$.

If $T^{\prime}(\lambda)$ is positive definite then we replace $S$ in the last paragraph by the matrix containing $\ell$ or $\tilde{\ell}$ eigenvectors of the generalized matrix eigenvalue problem

$$
V^{T} T\left(\sigma_{k}\right) V x=\mu V^{T} T^{\prime}\left(\sigma_{k}\right) V x
$$

corresponding to the $\ell$ or $\tilde{\ell}$ largest eigenvalues. This modification of the restart procedure can be motivated by the linear maxmin theory. $\lambda_{\ell}$ is an $\ell$-th eigenvalue and $x_{\ell}$ a corresponding eigenvector of problem (1) if and only if $\mu_{\ell}=0$ is the $\ell$-largest eigenvalue of the linear problem

$$
T\left(\lambda_{\ell}\right) x=\mu x
$$

and $x_{\ell}$ is a corresponding eigenvector.

If $\sigma_{k}$ is a good approximation to $\lambda_{\ell}$, then

$$
T\left(\lambda_{\ell}\right) \approx T\left(\sigma_{k}\right)-\eta T^{\prime}\left(\sigma_{k}\right)
$$

is a first order approximation, and we can approximate (1) by the generalized eigenproblem

$$
\left(T\left(\sigma_{k}\right)-\eta T^{\prime}\left(\sigma_{k}\right)\right) x_{\ell}=0 .
$$

The $\ell$ largest eigenvalue $\eta_{\ell}$ is near 0 , if $\sigma_{k}$ is a good approximation to $\lambda_{\ell}$, and it can be characterized by a maxmin principle

$$
0 \approx \eta_{\ell}=\max _{V \in S_{\ell}} \min _{v \in V \backslash\{0\}} \frac{v^{T} T\left(\sigma_{k}\right) v}{v^{T} T^{\prime}\left(\sigma_{k}\right) v} .
$$

The maximum is attained by the subspace spanned by the eigenvectors corresponding to the $\ell$ largest eigenvalues of (14) which motivates the choice of $S$.

\section{$5 \quad$ Numerical example}

To test our method we consider a mathematical model which describes the problem governing free vibrations of a tube bundle immersed in a slightly 
compressible fluid under the following simplifying assumptions: The tubes are assumed to be rigid, assembled in parallel inside the fluid, and elastically mounted in such a way that they can vibrate transversally, but they can not move in the direction perpendicular to their sections. The fluid is assumed to be contained in a cavity which is infinitely long, and each tube is supported by an independent system of springs (which simulates the specific elasticity of each tube). Due to these assumptions, three-dimensional effects are neglected, and so the problem can be studied in any transversal section of the cavity. Considering small vibrations of the fluid (and the tubes) around the state of rest, it can also be assumed that the fluid is irrotational.

Mathematically this problem can be described in the following way (cf. [7], [3]). Let $\Omega \subset \mathbb{R}^{2}$ (the section of the cavity) be an open bounded set with locally Lipschitz continuous boundary $\Gamma$. We assume that there exists a family $\Omega_{j} \neq \emptyset, j=1, \ldots, K$, (the sections of the tubes) of simply connected open sets such that $\bar{\Omega}_{j} \subset \Omega$ for every $j, \bar{\Omega}_{j} \cap \bar{\Omega}_{i}=\emptyset$ for $j \neq i$, and each $\Omega_{j}$ has a locally Lipschitz continuous boundary $\Gamma_{j}$. With these notations we set $\Omega_{0}:=\Omega \backslash \bigcup_{j=1}^{K} \Omega_{j}$. Then the boundary of $\Omega_{0}$ consists of $K+1$ connected components which are $\Gamma$ and $\Gamma_{j}, j=1, \ldots, K$.

We denote by $H^{1}\left(\Omega_{0}\right)=\left\{u \in L^{2}\left(\Omega_{0}\right): \nabla u \in L^{2}\left(\Omega_{0}\right)^{2}\right\}$ the standard Sobolev space equipped with the usual scalar product. Then the eigenfrequencies and the eigenmodes of the fluid-solid structure are governed by the following variational eigenvalue problem (cf. [7], [3])

Find $\lambda \in \mathbb{R}$ and $u \in H^{1}\left(\Omega_{0}\right)$ such that for every $v \in H^{1}\left(\Omega_{0}\right)$

$$
c^{2} \int_{\Omega_{0}} \nabla u \cdot \nabla v d x=\lambda \int_{\Omega_{0}} u v d x+\sum_{j=1}^{K} \frac{\lambda \rho_{0}}{k_{j}-\lambda m_{j}} \int_{\Gamma_{j}} u n d s \cdot \int_{\Gamma_{j}} v n d s .
$$

Here $u$ is the potential of the velocity of the fluid, $c$ denotes the speed of sound in the fluid, $\rho_{0}$ is the specific density of the fluid, $k_{j}$ represents the stiffness constant of the spring system supporting tube $j, m_{j}$ is the mass per unit length of the tube $j$, and $n$ is the outward unit normal on the boundary of $\Omega_{0}$.

We consider the rational eigenvalue problem (15) where $\Omega$ is the ellipse with center $(0,0)$ and length of semiaxes 8 and 4 , and $\Omega_{j}, j=1, \ldots, 9$ are circles with radius 0.3 and centers $(-4,-2),(0,-2),(4,-2),(-5,0),(0,0),(5,0)$, $(-4,2),(0,2)$ and $(4,2)$. We assume that all constants in problem (15) are equal to 1 .

Discretizing problem (15) by finite elements one gets a rational matrix eigen- 
value problem

$$
T(\lambda) x:=-A x+\lambda B x+\frac{\lambda}{1-\lambda} C x=0
$$

where $C$ collects the contributions of all tubes. $A, B$, and $C$ are symmetric matrices, $A$ and $C$ are positive semidefinite, and $B$ is positive definite. In our example the dimension is $n=36040$.

Problem (16) has 28 eigenvalues $\lambda_{1} \leq \ldots \leq \lambda_{28}$ in the interval $J_{1}=(0,1)$ and a large number of eigenvalues $\tilde{\lambda}_{11} \leq \tilde{\lambda}_{12} \leq \ldots$ in $(1, \infty), 20$ of which are contained in $J_{2}:=(1,3)$.

We determined the approximations to the eigenvalues $\lambda_{1}, \ldots, \lambda_{28} \in J_{1}$ without restarts. We terminated the iteration for an eigenvalue if the relative residual $\left\|T\left(\sigma_{k}\right) u_{k}\right\| /\left\|u_{k}\right\|$ was less than $10^{-12}$.

We solved the correction equations by preconditioned GMRES, and we terminated the GMRES iteration after at most 10 steps or if the initial residual was reduced by a factor $10^{-3}$. As preconditioner we chose the LU factorization of $T(\sigma)$ since this could be obtained quite inexpensively in our example. If GMRES was not able to obtain the required residual reduction by $10^{-3}$ within 5 steps the preconditioner was no longer good enough, and we replaced it by the LU factorization of $T\left(\sigma_{k}\right)$ with the current eigenvalue approximation $\sigma_{k}$ . Since some iterations are needed to gather information we applied this rule beginning with the computation of $\lambda_{3}$.

With these parameters the algorithm needed $16 \mathrm{LU}$ decompositions and 438 GMRES steps in total to determine all 28 eigenvalues in $J_{1}$. The dimension of the subspace $V$ grew only to 106 . The convergence history is given in Figure 1 .

We repeated the computation of the eigenvalues in $J_{1}$ now restarting if the dimension exceeded 40 and reducing it to $\min (10,3+$ \#of eigenvalue). This time the algorithm needed $13 \mathrm{LU}$ factorizations and 470 GMRES steps. The convergence history in Figure 2 is very similar to that one without restarts.

Finally we determined the eigenvalues in the interval $J_{2}:=(1,3)$. Since the smallest eigenvalue in this interval is an eleventh eigenvalue we needed a subspace of dimension 11 that approximates the invariant subspace of $T\left(\tilde{\lambda}_{11}\right)$ corresponding to the 11 largest eigenvalues to start the algorithm. Not knowing $\tilde{\lambda}_{11}$ we started with the 11 dimensional subspace $K x=\mu M x$ corresponding to the 11 smallest eigenvalues. In this case the algorithm needed $15 \mathrm{LU}$ factorizations and 340 GMRES steps. The convergence history is given in Figure 3 . 


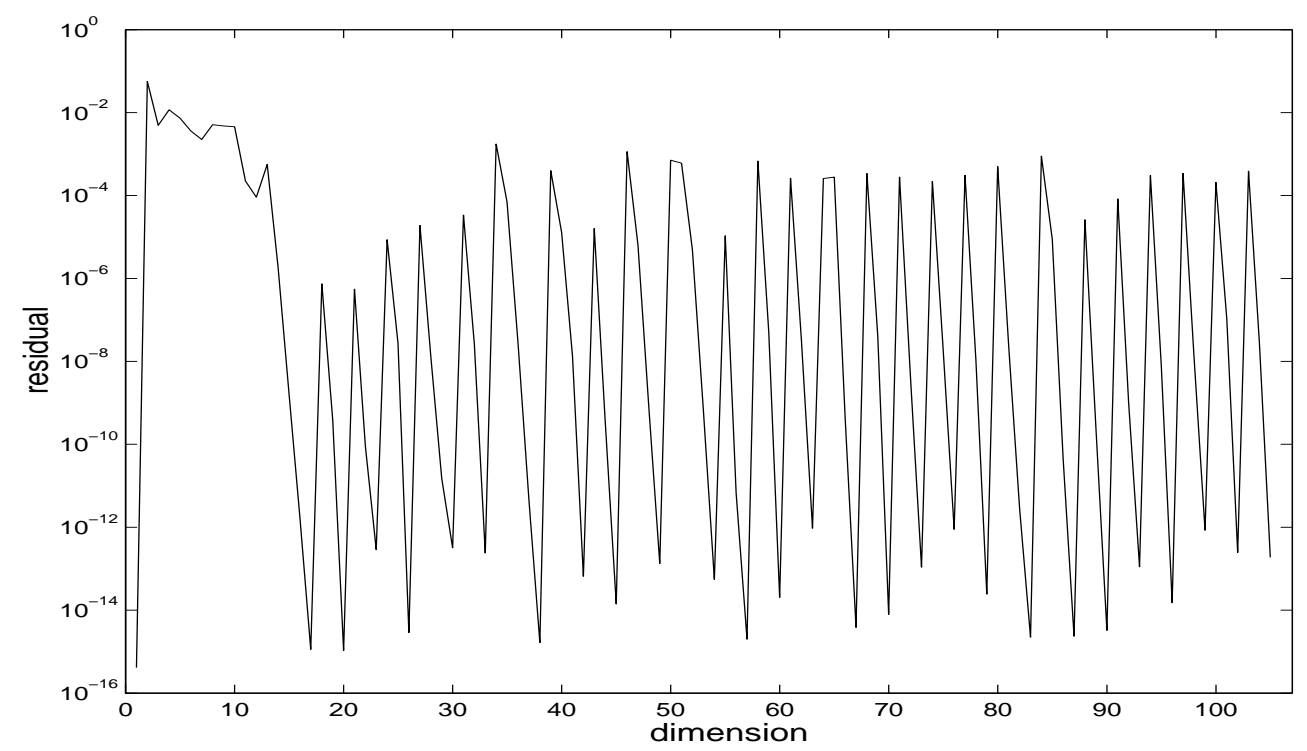

Fig. 1: Eigenvalues in $(0,1)$; no restarts

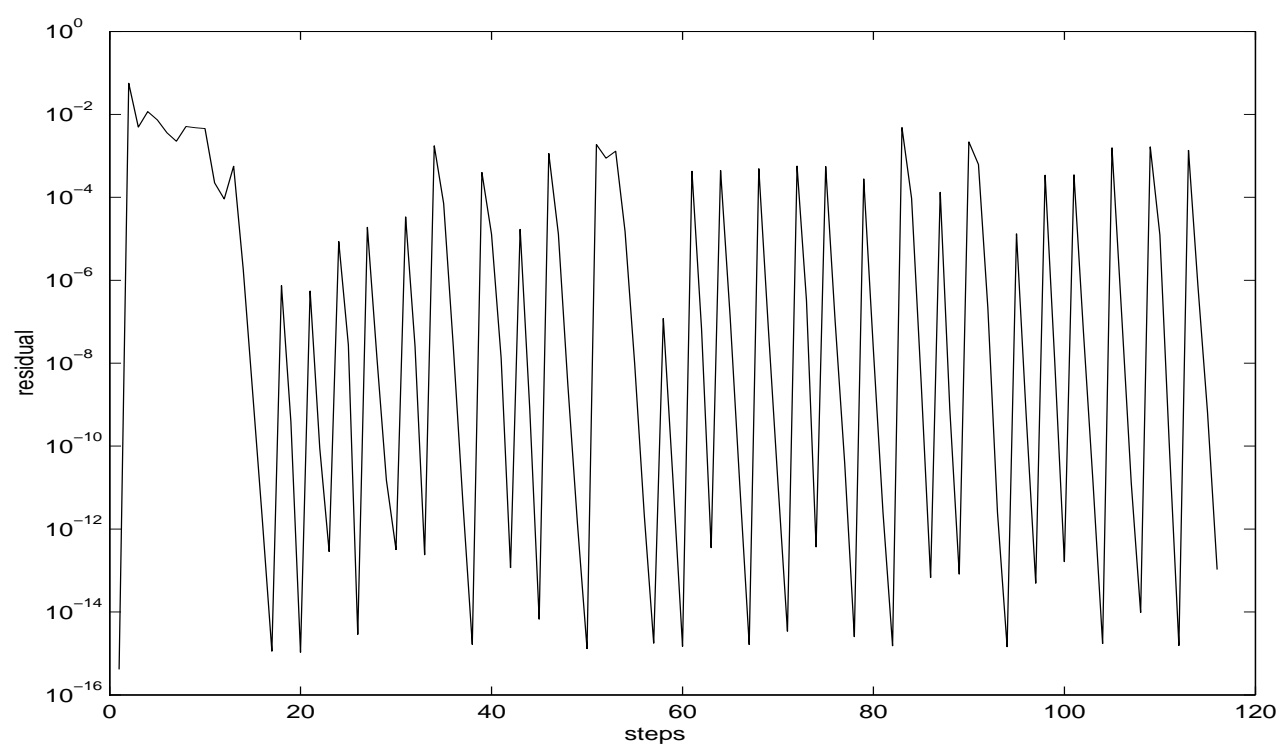

Fig. 2: Eigenvalues in $(0,1)$; restarted

\section{References}

[1] Bai, Z., Demmel, J., Dongarra, J., Ruhe, A., \& van der Vorst, H.A. (eds.), Templates for the Solution of Algebraic Eigenvalue Problems: A Practical Guide, SIAM, Philadelphia, 2000.

[2] Bai, Z., Sleijpen, G., \& van der Vorst H.A., Quadratic Eigenvalue Problems, Section 9.2 in [1], pp. $281-289$

[3] Conca, C., Planchard, J., \& Vanninathan, M, Existence and location of eigenvalues for fluid-solid structures, Comput.Meth.Appl.Mech.Engrg. 77 (1989) $253-291$ 


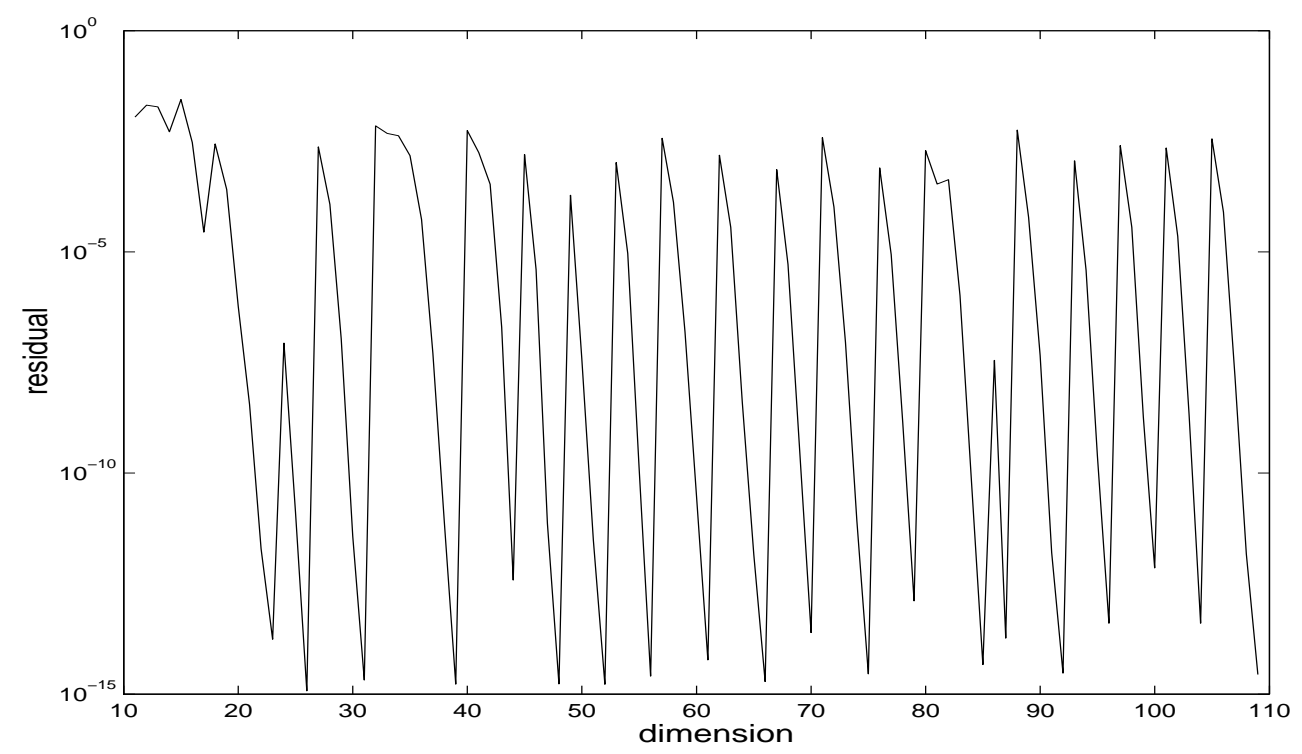

Fig. 3: Eigenvalues in $(1,3)$; no restarts

[4] Hager, P., Eigenfrequency Analysis. FE-Adaptivity and a Nonlinear Eigenvalue Problem, PhD thesis, Chalmers University of Technology, Göteborg 2001.

[5] Hager, P. \& Wiberg, N.E., The rational Krylov algorithm for nonlinear eigenvalue problems, in: Computational Mechanics for the Twenty-First Century, SaxeCoburg Publications, Edinburgh, 2000, pp. 379 - 402.

[6] Neumaier, A., Residual inverse iteration for the nonlinear eigenvalue problem, SIAM J. Numer. Anal. 22 (1985) $914-923$.

[7] Planchard, J., Eigenfrequencies of a tube bundle placed in a confined fluid, Comput.Meth.Appl.Mech.Engrg. 30 (1982) 75 - 93

[8] Ruhe, A., Algorithms for the nonlinear eigenvalue problem, SIAM J. Numer. Anal. 10 (1973) $674-689$.

[9] Ruhe, A., A rational Krylov algorithm for nonlinear matrix eigenvalue problems, Zapiski Nauchnych Seminarov POMI 268 (2000) 176 - 180.

[10] Sleijpen, G., \& van der Vorst, H.A., A Jacobi-Davidson iteration for linear eigenvalue problems, SIAM J. Matr. Anal. Appl. 17 (1996), $401-425$.

[11] Sleijpen, G., \& van der Vorst, H.A., The Jacobi-Davidson method for eigenproblems and its relation with accelerated inexact Newton schemes, in: Iterative Methods in Linear Algebra II, Proceedings of the Second IMACS International Symposium, North Holland, Amsterdam, 1996.

[12] Sleijpen, G., \& van der Vorst, H.A., Jacobi-Davidson Methods, Section 4.7 in [1], pp. $88-105$.

[13] Voss, H., Computation of eigenvalues of nonlinear eigenvalue problems, in: Proceedings of the Seventh South African Symposium on Numerical Mathematics, Durban, 1981, pp. $147-157$. 
[14] Voss, H., Solving a rational eigenvalue problem in fluid-structure interaction, To appear in Proceedings of the Seventh International Conference on Applications of High-Performance Computers in Engineering, Bologna, September 2002

[15] Voss, H., \& Werner, B., A minimax principle for nonlinear eigenvalue problems with application to nonoverdamped systems, Math.Meth.Appl.Sci. 4 (1982) 415 424 . 